\title{
Article \\ Spontaneous Lorentz Violation from Infrared Gravity
}

\author{
Fabrizio Illuminati ${ }^{1,2}$, Gaetano Lambiase ${ }^{2,3}$ (D) and Luciano Petruzziello ${ }^{1,2, *(D)}$ \\ 1 Dipartimento di Ingegneria Industriale, Università degli Studi di Salerno, Via Giovanni Paolo II, \\ 132, 84084 Fisciano, Italy; filluminati@unisa.it \\ 2 INFN, Sezione di Napoli, Gruppo Collegato di Salerno, Via Giovanni Paolo II, 132, 84084 Fisciano, Italy; \\ lambiase@sa.infn.it \\ 3 Dipartimento di Fisica, Università degli Studi di Salerno, Via Giovanni Paolo II, 132, 84084 Fisciano, Italy \\ * Correspondence: lupetruzziello@unisa.it
}

check for updates

Citation: Illuminati, F.; Lambiase, G.; Petruzziello, L. Spontaneous Lorentz Violation from Infrared Gravity. Symmetry 2021, 13, 1854. https:// doi.org/10.3390/sym13101854

Academic Editors: Chris Fields, Antonino Marciano and Jerzy Kowalski Glikman

Received: 7 July 2021

Accepted: 20 August 2021

Published: 3 October 2021

Publisher's Note: MDPI stays neutral with regard to jurisdictional claims in published maps and institutional affiliations.

Copyright: (c) 2021 by the authors. Licensee MDPI, Basel, Switzerland. This article is an open access article distributed under the terms and conditions of the Creative Commons Attribution (CC BY) license (https:// creativecommons.org/licenses/by/ $4.0 /)$.

\begin{abstract}
In this paper, we investigate a novel implication of the non-negligible spacetime curvature at large distances when its effects are expressed in terms of a suitably modified form of the Heisenberg uncertainty relations. Specifically, we establish a one-to-one correspondence between this modified uncertainty principle and the Standard Model Extension (SME), a string-theoretical effective field theory that accounts for both explicit and spontaneous breaking of Lorentz symmetry. This tight correspondence between string-derived effective field theory and modified quantum mechanics with extended uncertainty relations is validated by comparing the predictions concerning a deformed Hawking temperature derived from the two models. Moreover, starting from the experimental bounds on the gravity sector of the SME, we derive the most stringent constraint achieved so far on the value of the free parameter in the extended Heisenberg uncertainty principle.
\end{abstract}

Keywords: extended uncertainty principle; Standard Model Extension; Lorentz violation

\section{Introduction}

Since the establishment of quantum mechanics and general relativity, there has been a constantly growing effort to merge quantum and gravitational effects at arbitrary energy scales in a complete and consistent theoretical framework. This effort has produced several plausible candidates for a quantum theory of the gravitational interaction, such as string theory [1,2], loop quantum gravity [3,4], and doubly special relativity [5-7]. The study of the interplay between quantum and gravitational effects appears to be particularly important at the currently reachable energies, as this is the regime where we have hope that physical predictions can factually be probed in tabletop laboratory tests. Therefore, the analysis of low-energy, infrared (IR) quantum gravitational manifestations may represent a promising starting point for the possible construction of ultraviolet (UV) complete models of quantum gravity. In this respect, an additional motivation for the study of IR phenomena stems from the UV/IR duality discovered for the first time in the context of the AdS/CFT correspondence [8-10].

In the present paper, we focus on IR gravity to exhibit how Lorentz symmetry is affected by the non-negligible spacetime curvature at large distances. In a quantum mechanical setting, such a feature can be incorporated by extending the Heisenberg uncertainty principle (HUP) with the addition of a position-dependent correction that introduces a non-vanishing minimal uncertainty in momentum [11-15] and provides a form of an extended uncertainty principle (EUP). Among different versions of the EUP, the best known one includes a universal modification that is geometric-independent; indeed, such a generalization of the standard uncertainty relations arises naturally when merging quantum mechanics and general relativity. In other scenarios, the version of the EUP that is accounted for entails a dependence on the intrinsic geometric properties of the underlying background curvature; for more details along this direction, see Refs. [16-20]. For the 
sake of completeness, it is worth mentioning that several works inspired by string theory [21-24] also allow for the existence of a momentum-dependent correction to the HUP; such an extension is known as the generalized uncertainty principle (GUP) (for several development of this subject, see Refs. [25-38] and therein).

In order to quantify the breakdown of Lorentz symmetry induced by the EUP, we consider a string-theoretical effective field theory according to which any operator appearing in the Standard Model (SM) Lagrangian is contracted with Lorentz-violating fields [39,40]. This model is known as the Standard Model Extension (SME), and we will focus in particular on its gravity sector [41,42] in the non-relativistic limit [43]. Specifically, along the line of Ref. [44], in the following, we establish a one-to-one correspondence between the predictions of the EUP and of the SME concerning the deformation of the Hawking temperature for a Schwarzschild black hole:

$$
T_{H}=\frac{\hbar c^{3}}{8 \pi k_{B} G M}
$$

In so doing, we essentially relate the free deformation parameter arising in the framework of the EUP with the Lorentz-violating fields contained in the SME Lagrangian, thereby explaining spontaneous Lorentz symmetry breaking in terms of large-scale effects. Moreover, by relying on the experimental bounds associated with the SME gravity sector, we manage to derive novel constraints on the EUP free deformation parameter, which are more stringent than the previously known ones [15].

The paper is organized as follows: in Section 2, we briefly give an overlook of the main aspects of EUP together with a heuristic derivation of the modified Hawking temperature. The same procedure is carried out for the SME in Section 3, whereas Section 4 contains the theoretical and numerical comparison between the two predictions. Finally, Section 5 contains the concluding remarks and discussion.

\section{Extended Uncertainty Principle and Modified Hawking Temperature}

Starting from the Heisenberg uncertainty principle

$$
\Delta x \Delta p \geq \frac{\hbar}{2}
$$

one can incorporate the influence of spacetime curvature at large distances by adding a position-dependent term in the r.h.s. of Equation (2), namely [11,13-15]

$$
\Delta x \Delta p \geq \frac{\hbar}{2}\left(1+\alpha \Delta x^{2}\right)
$$

with $\alpha$ being the inverse of a squared length and $\alpha \Delta x^{2} \ll 1$. One possible interpretation of the free deformation parameter $\alpha$ is to conceive it as a function of the cosmological constant $\Lambda$ in a (anti-) de Sitter space [45]. However, in greater generality, it can simply be seen as a consequence of the intrinsic spacetime curvature at large cosmological distances that enforces a limit to the precision with which to resolve the momentum of a point particle [15]. In turn, this fact implies that there exists a minimal uncertainty for $p$ proportional to the constant $\alpha$, i.e., $\Delta p_{\text {min }} \simeq \hbar \sqrt{|\alpha|}$. Note that the parameter $\alpha$ does not have to be necessarily constant; indeed, it may be a function of spacetime position or even a stochastic variable. Similar considerations have already been addressed in the context of GUP [30,38] and they equally hold true for the EUP currently investigated.

The above inequality (3) can be straightforwardly derived from the deformed canonical commutation relation:

$$
[\hat{X}, \hat{P}]=i \hbar\left(\mathbf{1}+\alpha \hat{X}^{2}\right) .
$$


From the previous equation, one can deduce a simple representation of $\hat{X}$ and $\hat{P}$ in terms of auxiliary operators $\hat{x}$ and $\hat{p}$ for which the standard canonical commutation relation holds (i.e., $[\hat{x}, \hat{p}]=i \hbar)$. Specifically:

$$
\hat{X}=\hat{x}, \quad \hat{P}=\left(1+\alpha \hat{x}^{2}\right) \hat{p}-2 i \alpha \hbar \hat{x},
$$

where the second term in the r.h.s. of the second expression ensures that $\hat{P}=\hat{P}^{\dagger}$. The three-dimensional analysis of the above framework would allow for the emergence of spatial non-commutativity $[11,12]$, since one can immediately verify that $\left[\hat{X}_{j}, \hat{X}_{k}\right] \neq 0$. Nevertheless, we can safely ignore all the non-commutative corrections associated with the operators $\hat{X}_{j}$, as they would depend on higher powers of $\alpha$, i.e.,

$$
\hat{X}_{j}=\hat{x}_{j}+\mathcal{O}\left(\alpha^{2}\right),
$$

which, in the present, case are neglected. We refer to Refs. [11,12] for further mathematical details.

To evaluate the deformed Hawking temperature of a Schwarzschild black hole of mass $M$, we follow some simple heuristic considerations as outlined, e.g., in Refs. [27,46]. The starting point is the natural assumption that the position uncertainty of the photons just after they have been emitted by the black hole is proportional to the Schwarzschild radius, namely $\Delta x \simeq \gamma r_{s}$, with $\gamma$ to be fixed by requiring consistency with the standard picture in the limit $\alpha \rightarrow 0$. Under these circumstances, from Equation (3), we obtain

$$
\Delta p \simeq \frac{\hbar c^{2}}{4 \gamma G M}\left[1+4 \alpha \frac{G^{2} M^{2}}{c^{4}} \gamma^{2}\right] .
$$

Now, we can express the characteristic energy of the emitted photons $\Delta p c$ in terms of the temperature of the radiation in compliance with the equipartition theorem $[27,46]$, by virtue of which $\Delta p c \simeq k_{B} T$. Finally, the equation for the $\alpha$-deformed Hawking temperature $T_{E U P}$ reads

$$
T_{E U P}=\frac{\hbar c^{3}}{4 \gamma k_{B} G M}\left[1+4 \alpha \frac{G^{2} M^{2}}{c^{4}} \gamma^{2}\right],
$$

which, in terms of the Hawking temperature $T_{H}$, reads:

$$
T_{E U P}=T_{H}\left[1+16 \alpha \pi^{2} \frac{G^{2} M^{2}}{c^{4}}\right],
$$

where we have set $\gamma=2 \pi$ in order to recover the original Hawking result in the limit $\alpha \rightarrow 0$. For a thorough discussion on the above correspondence, we refer the reader to Ref. [47].

\section{Standard Model Extension and Modified Hawking Temperature}

The Standard Model Extension is a generalization of the Standard Model of particle physics, which predicts both explicit and spontaneous Lorentz symmetry breaking. Motivated by string-theoretical arguments [39,40], the SME enlarges the SM domain by contracting any SM field with Lorentz-violating operators that give rise to new phenomenology. Although the Standard Model Extension was originally conceived to extend the Standard Model only, later on, the gravitational interaction was also added, with the ensuing Lorentzviolating coefficients.

For our purposes, we are interested in investigating the minimal SME gravity sector [41-44], which includes exclusively Lorentz-violating operators of mass dimension 
three or four. In particular, by denoting with $S_{E H}$ and $S_{m}$ the Einstein-Hilbert and the matter action, respectively, the minimal SME total gravitational action reads [43]

$$
S=S_{E H}+S_{m}+S_{L V}, \quad S_{L V}=\frac{c^{4}}{16 \pi G} \int d^{4} x \sqrt{-g}\left(-u R+s^{\mu v} R_{\mu v}^{T}+t^{\mu v \rho \lambda} C_{\mu v \rho \lambda}\right),
$$

where $S_{L V}$ denotes the effective Lorentz symmetry-breaking action derived from string theory, $R$ is the Ricci scalar, $R_{\mu \nu}^{T}$ is the traceless Ricci tensor, $C_{\mu v \rho \lambda}$ is the Weyl conformal tensor, and $u, s^{\mu v}$ and $t^{\mu \nu \rho \lambda}$ are the Lorentz-violating effective fields.

In the regime of the post-Newtonian (PPN) approximation [48,49], a Schwarzschildlike solution of the linearized field equations for the minimal SME can be found [43], and it is given by

$$
d s^{2}=f(r) c^{2} d t^{2}-\frac{1}{f(r)} d r^{2}-r^{2} d \Omega^{2}
$$

where

$$
f(r)=1-\frac{2 G M}{r c^{2}}\left[1+\bar{s}^{i j} g_{i j}(\theta, \phi)\right] .
$$

In the above, $\bar{s}^{i j}$ denote the vacuum expectation values of the fields $s^{i j}$ and $g_{i j}(\theta, \phi)$ are functions of the angular coordinates, for which the inequality $g_{i j}(\theta, \phi) \leq 1$ holds regardless of the choice for $\theta$ and $\phi$. The absence of $\bar{u}$ in Equation (12) is related to the fact that a nonvanishing value for such parameter only amounts to a scaling of the PPN metric [43], and thus it can be set to zero. On the other hand, the disappearance of $\bar{t}^{\mu v \rho \lambda}$ is a typical feature occurring in the post-Newtonian SME gravitational phenomenology known as "t puzzle", and it has been extensively discussed in several works (see, for instance, Refs. [50-56]).

In order to evaluate the deformed Hawking temperature $T_{S M E}$ arising in the SME framework, we must compute $[44,57,58]$

$$
T_{S M E}=\left.\frac{\hbar c}{4 \pi k_{B}} \frac{d f(r)}{d r}\right|_{r=r_{0}},
$$

where $r_{0}$ solves the equation $f\left(r_{0}\right)=0$, thereby denoting the horizon radius. At this point, a straightforward calculation yields

$$
T_{S M E}=T_{H}\left[1-\bar{s}^{i j} g_{i j}(\theta, \phi)\right],
$$

which has to be compared with Equation (9) derived in the EUP framework. Before concluding this section, it is worth pointing out that the temperature (14) is anisotropic, as it explicitly depends on the angular position. However, such an observation does not undermine the validity of our main goal; indeed, Equation (9) might be anisotropic as well, since $\alpha$ is not bound to be a constant.

\section{Comparison and Consistency Conditions}

We will now look at the relations that are required in order to achieve consistency between the predictions (9) and (14) and thus relate the large-scale effects of spacetime curvature with spontaneous breaking of the Lorentz symmetry. The comparison of the deformed Hawking temperatures deduced from the two distinct physical settings shows that the two are consistent provided that the following identification holds:

$$
\alpha=-\frac{c^{4}}{16 \pi^{2} G^{2} M^{2}} \bar{s}^{i j} g_{i j}(\theta, \phi),
$$

for fixed values of $\theta$ and $\phi$. Therefore, as already argued, the magnitude of $\alpha$ is not constant, but it varies with the angular displacement, thus giving rise to an anisotropic $T$ also for the EUP-corrected Hawking temperature. Since the $g_{i j}(\theta, \phi)$ can always be taken as positive quantities [44], the sign of $\alpha$ strictly depends on the sign of the Lorentz-violating coefficients 
$\bar{s}^{i j}$. Additionally, by means of consistency arguments that do not require Lorentz invariance, it is known that $\alpha$ correctly characterizes an EUP associated with an expanding universe if and only if $\alpha<0[13,14,45]$. Therefore, to allow agreement with experimental evidence, assuming $g_{i j}(\theta, \phi) \simeq 1 \forall i, j[44]$, we must necessarily impose

$$
\sum_{i, j} \bar{s}^{i j}>0
$$

which realizes a novel bound on the admissible values of the Lorentz-violating coefficients. Of course, by virtue of Equation (15), the bounds holding for the quantities $\bar{s}^{i j}$ in turn imply bounds on the EUP free deformation parameter that lead to an extremely significant improvement with respect to the existing constraints [15] on the possible values of $\alpha$.

In Table 1 , we report the known bounds on $\bar{s}^{i j}$, the ensuing constraints that they entail on $\sqrt{|\alpha|}$ by virtue of Equation (15) and the corresponding experimental frameworks used to determine each bound.

Table 1. Estimated bounds on the EUP parameter.

\begin{tabular}{|c|c|c|}
\hline Experiments & Bounds on $\left|\bar{s}^{i j}\right|$ & Bounds on $\sqrt{|\alpha|}$ \\
\hline $\begin{array}{l}\text { Geodetic effect } \\
\left(M=M_{\oplus}\right)[59]\end{array}$ & $\left|\bar{s}^{i j}\right| \lesssim 10^{-3}$ & $\sqrt{|\alpha|} \lesssim 6.37 \times 10^{-2} \mathrm{~m}^{-1}$ \\
\hline $\begin{array}{l}\text { Gravity Probe B } \\
\left(M=M_{\oplus}\right)[59]\end{array}$ & $\left|\bar{s}^{i j}\right| \lesssim 10^{-4}$ & $\sqrt{|\alpha|} \lesssim 2.01 \times 10^{-2} \mathrm{~m}^{-1}$ \\
\hline $\begin{array}{l}\text { Frame dragging } \\
\left(M=M_{\oplus}\right)[59]\end{array}$ & $\left|\bar{s}^{i j}\right| \lesssim 10^{-7}$ & $\sqrt{|\alpha|} \lesssim 6.37 \times 10^{-4} \mathrm{~m}^{-1}$ \\
\hline Gravimetry $\left(M=M_{\oplus}\right)$ [59] & $\left|\bar{s}^{i j}\right| \lesssim 10^{-10}$ & $\sqrt{|\alpha|} \lesssim 2.01 \times 10^{-5} \mathrm{~m}^{-1}$ \\
\hline $\begin{array}{l}\text { Lunar laser ranging } \\
\left(M=M_{\oplus}\right)[59,60]\end{array}$ & $\left|\bar{s}^{i j}\right| \lesssim 10^{-12}$ & $\sqrt{|\alpha|} \lesssim 2.01 \times 10^{-6} \mathrm{~m}^{-1}$ \\
\hline $\begin{array}{l}\text { Torsion pendulum } \\
\left(M=M_{\oplus}\right)[59]\end{array}$ & $\left|\bar{s}^{i j}\right| \lesssim 10^{-15}$ & $\sqrt{|\alpha|} \lesssim 6.37 \times 10^{-8} \mathrm{~m}^{-1}$ \\
\hline $\begin{array}{l}\text { Perihelion precession } \\
\qquad\left(M=M_{\odot}\right)[59]\end{array}$ & $\left|\bar{s}^{i j}\right| \lesssim 10^{-9}$ & $\sqrt{|\alpha|} \lesssim 1.98 \times 10^{-10} \mathrm{~m}^{-1}$ \\
\hline $\begin{array}{c}\text { Binary pulsar } \\
\left(M=2.8 M_{\odot}\right)[59,61]\end{array}$ & $\left|\bar{s}^{i j}\right| \lesssim 10^{-11}$ & $\sqrt{|\alpha|} \lesssim 7.05 \times 10^{-12} \mathrm{~m}^{-1}$ \\
\hline $\begin{array}{l}\text { Solar-spin precession } \\
\qquad\left(M=M_{\odot}\right)[59]\end{array}$ & $\left|\bar{s}^{i j}\right| \lesssim 10^{-13}$ & $\sqrt{|\alpha|} \lesssim 1.98 \times 10^{-12} \mathrm{~m}^{-1}$ \\
\hline
\end{tabular}

The bounds on $\sqrt{|\alpha|}$ derived from the geodetic effect and Gravity Probe B are similar to the ones typically encountered in phenomenological works on this topic [15]. Consequently, we note that all the other results contained in Table 1 considerably strengthen the constraint on $\alpha$. Specifically, the inequality $\sqrt{|\alpha|} \lesssim 1.98 \times 10^{-12} \mathrm{~m}^{-1}$ provides the best current bound on the EUP free deformation parameter $\alpha$, and further refinement of the experimental sensitivity may allow for an even more stringent constraint.

It is worth observing that, should one regard $\alpha$ as emergent from a non-vanishing cosmological constant in de Sitter space, we would have $\alpha=-\Lambda / 3 \simeq-3.66 \times 10^{-53} \mathrm{~m}^{-2}$, which, in the framework of a near-Earth experiment, would correspond to $\left|\bar{s}^{i j}\right| \simeq 9.06 \times 10^{-54}$. This is in line with the expectation that Lorentz-violating coefficients should be indeed extremely small corrections to Lorentz-symmetric physics [43].

\section{Concluding Remarks}

In this work, we have investigated the consequences of relating large-scale effects ascribable to the non-negligible spacetime curvature and the spontaneous Lorentz symmetry breaking as described by the gravity sector of the Standard Model Extension. The relation is obtained by requiring consistency between the different modifications of the Hawking temperature predicted by the SME and by a quantum mechanical model endowed with 
an extended uncertainty principle deformed due to spacetime curvature effects. Investigating the consequences of the consistency relations imposed between the SME and the EUP, we have shown how to significantly enhance the existing bounds on the EUP curvature-induced deformation parameter starting from the experimental constraints on the Lorentz-violating coefficients that enter the gravity sector of the SME. This simple comparison points at the possibility, in suitable settings, of probing high-energy quantum physics via low-energy gravitational effects. To some extent, the idea underlying the present study shares the same philosophy characterizing the well-known AdS/CFT correspondence [8], as it may potentially provide some further insight towards a full understanding of the UV/IR duality.

Author Contributions: L.P. performed calculations and drafted the paper. F.I. and G.L. supervised the work. All authors discussed the results and edited the paper. All authors have read and agreed to the published version of the manuscript.

Funding: This research received no external funding.

Institutional Review Board Statement: Not applicable.

Informed Consent Statement: Not applicable.

Data Availability Statement: Not applicable.

Conflicts of Interest: The authors declare no conflict of interest.

\section{References}

1. Polchinski, J. String Theory; Cambridge University Press: Cambridge, MA, USA, 2011.

2. Green, M.B.; Schwarz, J.H.; Witten, E. Superstring Theory; Cambridge University Press: Cambridge, MA, USA, 2012.

3. Rovelli, C. Quantum Gravity; Cambridge University Press: Cambridge, MA, USA, 2010.

4. Rovelli, C.; Vidotto, F. Covariant Loop Quantum Gravity; Cambridge University Press: Cambridge, MA, USA, 2015.

5. Amelino-Camelia, G.; Ellis, J.R.; Mavromatos, N.E.; Nanopoulos, D.V.; Sarkar, S. Tests of quantum gravity from observations of gamma-ray bursts. Nature 1998, 393, 763. [CrossRef]

6. Magueijo, J.; Smolin, L. Lorentz invariance with an invariant energy scale. Phys. Rev. Lett. 2002, 88, 190403. [CrossRef] [PubMed]

7. Amelino-Camelia, G. Doubly special relativity. Nature 2002, 418, 34. [CrossRef] [PubMed]

8. Maldacena, J.M. The Large N limit of superconformal field theories and supergravity. Adv. Theor. Math. Phys. 1998, 2, 231. [CrossRef]

9. Witten, E. Anti-de Sitter space and holography. Adv. Theor. Math. Phys. 1998, 2, 253. [CrossRef]

10. Susskind, L.; Witten, E. The Holographic bound in anti-de Sitter space. arXiv 1998, arXiv:9805114.

11. Hinrichsen, H.; Kempf, A. Maximal localization in the presence of minimal uncertainties in positions and momenta. J. Math. Phys. 1996, 37, 2121. [CrossRef]

12. Kempf, A. On quantum field theory with nonzero minimal uncertainties in positions and momenta. J. Math. Phys. 1997, $38,1347$. [CrossRef]

13. Filho, R.N.C.; Braga, J.P.M.; Lira, J.H.S.; Andrade, J.S. Extended uncertainty from first principles. Phys. Lett. B 2016, $755,367$. [CrossRef]

14. Ong, Y.C.; Yao, Y. Generalized Uncertainty Principle and White Dwarfs Redux: How Cosmological Constant Protects Chandrasekhar Limit. Phys. Rev. D 2018, 98, 126018. [CrossRef]

15. Zarei, M.; Mirza, B. Minimal Uncertainty in Momentum: The Effects of IR Gravity on Quantum Mechanics. Phys. Rev. D 2009, 79, 125007. [CrossRef]

16. Schurmann, T.; Hoffmann, I. A closer look at the uncertainty relation of position and momentum. Found. Phys. 2009, 39, 958. [CrossRef]

17. Schürmann, T. Uncertainty principle on 3-dimensional manifolds of constant curvature. Found. Phys. 2018, 48, 716. [CrossRef]

18. Dabrowski, M.P.; Wagner, F. Extended Uncertainty Principle for Rindler and cosmological horizons. Eur. Phys. J. C 2019, $79,716$. [CrossRef]

19. Dabrowski, M.P.; Wagner, F. Asymptotic Generalized Extended Uncertainty Principle. Eur. Phys. J. C 2020, 80, 676. [CrossRef]

20. Petruzziello, L.; Wagner, F. Gravitationally induced uncertainty relations in curved backgrounds. Phys. Rev. D 2021, $103,104061$. [CrossRef]

21. Amati, D.; Ciafaloni, M.; Veneziano, G. Superstring collisions at planckian energies. Phys. Lett. B 1987, 197, 81. [CrossRef]

22. Kempf, A.; Mangano, G.; Mann, R.B. Hilbert space representation of the minimal length uncertainty relation. Phys. Rev. D 1995, 52, 1108. [CrossRef]

23. Maggiore, M. A Generalized Uncertainty Principle in Quantum Gravity. Phys. Lett. B 1993, 304, 65. [CrossRef]

24. Adler, R.J.; Santiago, D.I. On gravity and the uncertainty principle. Mod. Phys. Lett. A 1999, 14, 1371. [CrossRef] 
25. Kanazawa, T.; Lambiase, G.; Vilasi, G.; Yoshioka, A. Noncommutative Schwarzschild geometry and generalized uncertainty principle. Eur. Phys. J. C 2019, 79, 95. [CrossRef]

26. Luciano, G.G.; Petruzziello, L. GUP parameter from Maximal Acceleration. Eur. Phys. J. C 2019, 79, 283. [CrossRef]

27. Adler, R.J.; Chen, P.; Santiago, D.I. The Generalized Uncertainty Principle and Black Hole Remnants. Gen. Rel. Grav. 2001, 33, 2101. [CrossRef]

28. Chen, P.; Ong, Y.C.; Yeom, D. Black hole remnants and the information loss paradox. Phys. Rep. 2015, 603, 1. [CrossRef]

29. Scardigli, F.; Lambiase, G.; Vagenas, E. GUP parameter from quantum corrections to the Newtonian potential. Phys. Lett. B 2017, 767, 242. [CrossRef]

30. Chen, P.; Ong, Y.C.; Yeom, D.H. Generalized Uncertainty Principle: Implications for Black Hole Complementarity. J. High Energy Phys. 2014, 12, 021. [CrossRef]

31. Buoninfante, L.; Luciano, G.G.; Petruzziello, L. Generalized Uncertainty Principle and Corpuscular Gravity. Eur. Phys. J. C 2019, 79, 663. [CrossRef]

32. Buoninfante, L.; Lambiase, G.; Luciano, G.G.; Petruzziello, L. Phenomenology of GUP stars. Eur. Phys. J. C 2020, 80, 853. [CrossRef]

33. Petruzziello, L. Generalized uncertainty principle with maximal observable momentum and no minimal length indeterminacy. Class. Quant. Grav. 2021, 38, 135005. [CrossRef]

34. Hossenfelder, S. Minimal Length Scale Scenarios for Quantum Gravity. Living Rev. Rel. 2013, 16, 2. [CrossRef] [PubMed]

35. Blasone, M.; Lambiase, G.; Luciano, G.G.; Petruzziello, L.; Scardigli, F. Heuristic derivation of Casimir effect in minimal length theories. Int. J. Mod. Phys. D 2020, 29, 2050011. [CrossRef]

36. Kumar, S.P.; Plenio, M.B. On Quantum Gravity Tests with Composite Particles. Nat. Commun. 2020, 11, 3900. [CrossRef] [PubMed]

37. Das, S.; Vagenas, E.C. Universality of Quantum Gravity Corrections. Phys. Rev. Lett. 2008, 101, 221301. [CrossRef]

38. Petruzziello, L.; Illuminati, F. Quantum gravitational decoherence from fluctuating minimal length and deformation parameter at the Planck scale. Nat. Commun. 2021, 12, 4449. [CrossRef] [PubMed]

39. Colladay, D.; Kostelecky, V.A. CPT violation and the standard model. Phys. Rev. D 1997, 55, 6760. [CrossRef]

40. Colladay, D.; Kostelecky, V.A. Lorentz violating extension of the standard model. Phys. Rev. D 1998, 58, 116002. [CrossRef]

41. Kostelecky, V.A. Gravity, Lorentz violation, and the standard model. Phys. Rev. D 2004, 69, 105009. [CrossRef]

42. Bluhm, R.; Kostelecky, V.A. Spontaneous Lorentz violation, Nambu-Goldstone modes, and gravity. Phys. Rev. D 2006, 71, 065008. [CrossRef]

43. Bailey, Q.G.; Kostelecky, V.A. Signals for Lorentz violation in post-Newtonian gravity. Phys. Rev. D 2006, 74, 045001. [CrossRef]

44. Lambiase, G.; Scardigli, F. Lorentz violation and generalized uncertainty principle. Phys. Rev. D 2018, 97, 075003. [CrossRef]

45. Mignemi, S. Extended uncertainty principle and the geometry of (anti)-de Sitter space. Mod. Phys. Lett. A 2010, $25,1697$. [CrossRef]

46. Scardigli, F. Some heuristic semiclassical derivations of the Planck length, the Hawking effect and the Unruh effect. Nuovo Cim. B 1995, 110, 1029. [CrossRef]

47. Chung, W.S.; Hassanabadi, H. Black hole temperature and Unruh effect from the extended uncertainty principle. Phys. Lett. B 2019, 793, 451. [CrossRef]

48. Weinberg, S. Gravitation and Cosmology; John Wiley \& Sons, Inc.: New York, NY, USA, 1972.

49. Will, C.M. The Confrontation between General Relativity and Experiment. Living Rev. Rel. 2014, 17, 4. [CrossRef]

50. Bailey, Q.G. Time delay and Doppler tests of the Lorentz symmetry of gravity. Phys. Rev. D 2009, 80, 044004. [CrossRef]

51. Bailey, Q.G. Lorentz-violating gravitoelectromagnetism. Phys. Rev. D 2010, 82, 065012. [CrossRef]

52. Altschul, B.; Bailey, Q.G.; Kostelecký, V.A. Lorentz violation with an antisymmetric tensor. Phys. Rev. D 2010, 81, 065028. [CrossRef]

53. Tso, R.; Bailey, Q.G. Light-bending tests of Lorentz invariance. Phys. Rev. D 2011, 84, 085025. [CrossRef]

54. Tasson, J.D. Lorentz violation, gravitomagnetism, and intrinsic spin. Phys. Rev. D 2012, 86, 124021. [CrossRef]

55. Bailey, Q.G.; Kostelecký, V.A.; Xu, R. Short-range gravity and Lorentz violation. Phys. Rev. D 2015, 91, 022006. [CrossRef]

56. Bonder, Y. Lorentz violation in the gravity sector: The t puzzle. Phys. Rev. D 2015, 91, 125002. [CrossRef]

57. Zee, A. Quantum Field Theory in a Nutshell; Princeton University Press: Princeton, NJ, USA, 2003.

58. Scardigli, F.; Casadio, R. Gravitational tests of the Generalized Uncertainty Principle. Eur. Phys. J. C 2015, 75, 425. [CrossRef]

59. Kostelecky, V.A.; Russell, N. Data Tables for Lorentz and CPT Violation. Rev. Mod. Phys. 2011, 83, 11. [CrossRef]

60. Bourgoin, A.; Hees, A.; Bouquillon, S.; Poncin-Lafitte, C.L.; Francou, G.; Angonin, M.C. Testing Lorentz symmetry with Lunar Laser Ranging. Phys. Rev. Lett. 2016, 117, 241301. [CrossRef]

61. Shao, L. Tests of Local Lorentz Invariance Violation of Gravity in the Standard Model Extension with Pulsars. Phys. Rev. Lett. 2014, 112, 111103. [CrossRef] 